\title{
Development of a new model for acute myocardial infarction in rabbits
}

\author{
Mei-Yun TAN ${ }^{1) \#}$, Bo XIA ${ }^{1) \#, ~ Z h u n ~ X I A O ~}{ }^{2) \#}$, Zhong-Wei FAN ${ }^{1)}$, Hong ZHOU ${ }^{3)}$, \\ Xing $\mathrm{GUO}^{3)^{*}}$ and Yong-Can HUANG ${ }^{4,5,6)}$ * \\ 1)Department of Bone and Joint Surgery, The Affiliated Hospital of Southwest Medical University, Luzhou \\ 646000, China \\ ${ }^{2)}$ Reproductive Medical Center of West China 2nd Hospital, Sichuan University, Chengdu, Sichuan Province \\ 610041, China \\ ${ }^{3)}$ Department of Burns and Plastic Surgery, The Affiliated Hospital of Southwest Medical University, Luzhou \\ 646000, China \\ 4)Department of Orthopaedics and Traumatology, Li Ka Shing Faculty of Medicine, The University of Hong \\ Kong, Hong Kong SAR 999077, China \\ ${ }^{5)}$ Shenzhen Key Laboratory of Spine Surgery, Department of Spine Surgery, Peking University Shenzhen \\ Hospital, Shenzhen 518036, China \\ ${ }^{6)}$ Shenzhen Engineering Laboratory of Orthopaedic Regenerative Technologies, Orthopaedic Research Center, \\ Peking University Shenzhen Hospital, Shenzhen 518036, China
}

\section{J. Vet. Med. Sci.}

79(3): 467-473, 2017

doi: 10.1292/jvms.16-0114

Received: 11 March 2016

Accepted: 26 December 2016

Published online in J-STAGE:

21 January 2017
ABSTRACT. The rabbit left anterior descending coronary artery is not macroscopically apparent; this often leads to failure in creation of an acute myocardial infarction (AMI) model. In order to devise a simple method with good reproducibility and high success rate for use as a rabbit AMI model, a new surgical technique was developed, in which the obtuse marginal (OM) branch of the left circumflex coronary artery was coagulated with an electric knife using a left parasternal approach. Four weeks after OM branch coagulation, an electrocardiogram (ECG), blood biochemistry analysis, echocardiographic measurements and pathologic analysis were performed. The left parasternal approach provided the surgeon clear visualization of the targeted blood vessel to accurately identify the proper site to occlude. The successful development of AMI was confirmed by ST segment elevation on the ECG, by high levels of AMI-related markers in blood samples, by cardiac functional damage reflected on echocardiographic images and by changes in pathological sections. Furthermore, an acceptable success rate and low mortality were achieved. Hence, this surgical technique was suggested to be a highly reliable and reproducible method to induce AMI in rabbits for the assessment of new therapeutic interventions or regenerative approaches.

KEY WORDS: acute myocardial infarction, left circumflex coronary artery, left parasternal approach, obtuse marginal branch

Acute myocardial infarction (AMI) is one of the leading causes of death and disability worldwide [24]. Biological therapies for AMI-related diseases, such as stem cell- and biomaterial-based approaches have gained increasing interest [3, 17, 18, 26], and preclinical animal models are thus essential for study of AMI-related pathophysiology in humans and to evaluate the safety and effectiveness of promising treatments.

Closed-chest modeling methods have been used to create AMI in several species of animals [16, 19]. However, as these modeling methods require dedicated facilities and highly trained surgeons, the models are logistically complex and costly. Ligation of the left anterior descending coronary artery (LAD) after thoracotomy remains a relatively simple and economical method for AMI animal model development, especially in rodents and rabbits. However, the LAD in rabbits is buried in the heart muscle, and the surface of the epicardium is covered by massive fat tissue [25]. Thus, the course of the rabbit LAD is difficult to identify macroscopically, and the ideal ligation location is difficult to determine for successful AMI model development. Therefore, an easy and reproducible technique for the development of AMI in rabbits is required.

As described in previous literature [9], it was found that the obtuse marginal (OM) branch of the left circumflex coronary artery, along the left rim of the heart, was thick, long and superficial, and its surface was not covered by fat tissue. It was thus proposed

*Correspondence to: Guo, X., No.25 Taiping Street, Luzhou, Sichuan 64600o, China. e-mail: gx412@126.com, Huang, Y.-C., Rm914, Lab Block, 21 Sassoon Road, Pokfulam, Hong Kong. e-mail: y.c.huang@connect.hku.hk

\#These three authors contributed equally to this work.

(O2017 The Japanese Society of Veterinary Science

This is an open-access article distributed under the terms of the Creative Commons Attribution Non-Commercial No Derivatives (by-nc-nd) License. (CC-BY-NC-ND 4.0: https://creativecommons.org/licenses/by-nc-nd/4.0/) 
that the clear and direct visualization of the OM branch might ensure the accurate identification of the ideal ligation site, thereby ensuring the successful development of an AMI model in rabbits.

Hence, in this study, it was hypothesized that coagulation of the OM branch would be a very easy and highly reproducible method for inducing AMI in rabbits. To test this hypothesis, OM branch coagulation was performed in New Zealand White rabbits using the left parasternal approach. At 4 weeks after OM branch coagulation, electrocardiographic, blood biochemical, echocardiographic and pathologic analyses were conducted.

\section{MATERIALS AND METHODS}

\section{Animals}

Thirty-five male New Zealand White rabbits (3 months old, 1.5-2 kg) were purchased from the Laboratory Animal Center of Southwest Medical University. All animal procedures were performed according to the National Institutes of Health Guide for the Care and Use of Laboratory Animals (NIH Pub. No. 86-23, Revised 1996). All animal procedures were performed following protocols approved by the Animal Care and Use Committees of Southwest Medical University.

\section{Instruments}

The monopolar GD350-D high-frequency electric knife used in our experiment was made by Shanghai Hutong Electric Instrument Factory (Shanghai, China). To detach the sternocostal joints, we set the instrument at electric cut mode, level " 2 ," $30 \mathrm{~W}$. To coagulate the OM branch and stop bleeding at the incision, we set the instrument at electric coagulation mode, level "1," 35 W.

\section{Surgical approach for OM branch exposure}

All animals were anesthetized with an intramuscular injection of ketamine (35 mg/kg) and xylazine (5 mg/kg) to the left thigh. The animals were placed in the supine position on a heating pad and maintained at a temperature of $38.0-39.0^{\circ} \mathrm{C}$. The hair covering the chest and limbs was removed by wiping with $8 \% \mathrm{Na}_{2} \mathrm{~S}$ aqueous solution. Then, standard electrocardiography (ECG) was monitored continuously during the surgical procedure. For local anesthesia, $20 \mathrm{mg} / \mathrm{kg}$ lidocaine was injected into the subcutaneous area overlying the sternocostal joints. All rabbits were subjected to left parasternal sternotomy. Under sterile conditions, an incision was made with a conventional scalpel along the left side of the sternum. The sternocostal joints starting at the xiphoid process plane and ending at the jugular notch plane were detached carefully with an electric knife. Then, the sternocostal joints were gently pulled open with a retractor for adequate exposure of the operating region. The pericardium was incised with a small pair of scissors to expose the left myocardial ventricular wall. After removal of the pericardium, the OM branch was visualized under a shadow less lamp.

\section{Coagulation of the OM branch for AMI}

After setting the electric knife to coagulation mode, the OM branch was coagulated by direct macroscopic contact. The contact point was precisely placed at a location two-thirds of the distance from the bottom of the heart to the left ventricular apex (Fig. 1), and a coagulating time of $1 \mathrm{sec}$ was essential to avoid direct injury to the myocardium and conduction system of the heart. For sham animals, the pericardium was incised without OM branch coagulation. After adequate incisional hemostasis with the electric knife, the thorax was closed with interrupted 4-0 nylon sutures. To protect the rabbits in both groups from incisional infection, intramuscular injections of $25 \mathrm{mg} / \mathrm{kg}$ cefuroxime were given to the left thigh at $30 \mathrm{~min}$ before surgery and 24,48 and $72 \mathrm{hr}$ after surgery.

\section{Blood biochemistry analysis}

As myoglobin, the MB isoenzyme of creatine kinase (CK-MB) and high-sensitivity troponin I (hs-TnI) are released into the circulation when cardiac cell necrosis has occurred, they are specific for the diagnosis of AMI [15]. After AMI induction, blood samples were collected from the ear vein of each rabbit. Specifically, $3 \mathrm{ml}$ of blood was collected from each rabbit at $12 \mathrm{hr}$ after AMI induction for myoglobin assay; another $3 \mathrm{ml}$ blood was collected at $24 \mathrm{hr}$ after AMI induction for CK-MB assay and hs-TnI assay. Levels of these myocardial infarction-related markers were measured on an Abbott AxSYM analyzer (Abbott, Wiesbaden, Germany).

\section{Functional assessment by echocardiography}

Prior to and 4 weeks after OM branch coagulation, animals were sedated with an intramuscular injection of ketamine (10 mg/kg) and xylazine $(3 \mathrm{mg} / \mathrm{kg}$ ) to the left thigh. The chest was once again dehaired using the method described above. Two-dimensional and M-mode echocardiography was performed through the parasternal short axis using a 6-MHz transducer (Acuson Sequoia 512). The detection index included end-diastolic volume (EDV), end-systolic volume (ESV) and left ventricular ejection fraction (LVEF). All measurements were averaged on 5 consecutive cardiac cycles and performed by an experienced echocardiographer who was blinded to the animal groups and protocols.

\section{Pathological analysis}

Following echocardiographic measurements at 4 weeks after OM branch coagulation, the rabbits were sacrificed with an intravenous injection of ketamine $(600 \mathrm{mg})$. After the hearts were harvested, they were perfused with physiologic saline in a 

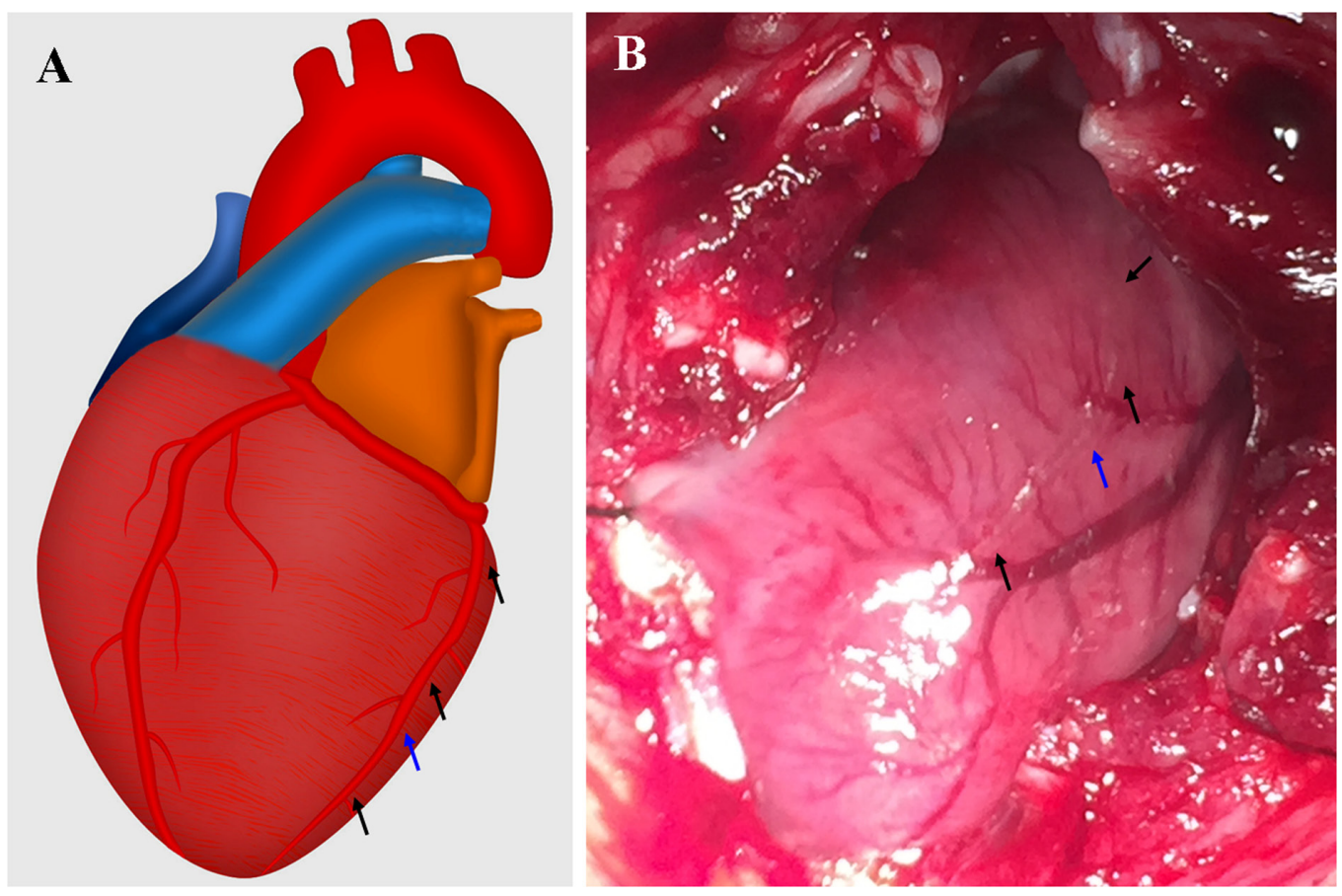

Fig. 1. Schematic (A) and physical (B) representation of obtuse marginal branch of the left circumflex coronary artery in the rabbit. The OM branch was thick, long, superficial and readily visible. Black arrows indicate the OM branch. Blue arrow indicates the coagulation site located at a point two-thirds from the bottom of the heart to the left ventricular apex.

retrograde manner. Following macroscopic examination, 3-mm thick sections were taken by transverse slicing through the center of visibly pale tan post-infarct fibrotic areas. Slices were fixed in 4\% formalin and embedded in paraffin. Sections, 5- $\mu \mathrm{m}$ thick, were stained with hematoxylin and eosin (HE) and Masson's trichrome. The blue and pale tissue in sections that stained with Masson's trichrome was regarded as infarcted myocardium. The infarct size, the left ventricular lateral wall thickness (LVLWT) and the total left ventricular cross-sectional area (LVCSA) were determined planimetrically using Image-Pro Plus 6.0 (Media Cybernatics, Rockville, MD, U.S.A.). The infarct size was expressed as the ratio of blue and pale area to the total LVCSA [10].

\section{Statistical analysis}

Data are expressed as mean \pm standard deviation. Analysis of data was performed using an unpaired $t$-test (SPSS 19.0 Inc., Chicago, IL, U.S.A.). $P<0.05$ (two-tailed) was considered statistically significant.

\section{RESULTS}

Of the 35 study animals, 2 died during initial anesthesia (5.7\%). The remaining 33 rabbits were randomly divided into two groups: the OM branch coagulation group $(n=23)$ and the sham group $(n=10)$. Five rabbits died in the OM branch coagulation group (overall mortality of 21.7\%): 2 died immediately after the operation due to ventricular fibrillation, 2 died 4-5 days after AMI induction due to progressive cardiogenic shock, and 1 died 2 days after the operation due to unknown causes. One rabbit in the sham group died from sepsis. Other animals in the OM branch coagulation group $(n=18)$ and sham group ( $n=9)$ survived to the end of follow-up. The AMI model was successfully established in all surviving animals in the OM branch coagulation group (100\%), as confirmed by ECG, blood biochemistry, echocardiography and pathology. The average time to identify the ideal ligation point in every rabbit was less than $1 \mathrm{~min}$. Meanwhile, the entire AMI modeling procedure time from anesthesia to the end of the procedure (skin suturing) was $24.2 \pm 5.7 \mathrm{~min}$. Bleeding of intercostal vessels was stopped effectively with the electric knife, and the average volume of incisional bleeding was about $3 \mathrm{~m} l(2 \mathrm{ml}-5 \mathrm{~m} l)$.

In all cases, ST segment elevation on ECG (Fig. 2), i.e., a typical change in AMI, was seen in less than 5 min after OM branch coagulation, but elevation was never observed in the sham group during the experimental period. Additionally, the level of AMIrelated markers (hs-TnI, CK-MB and $\mathrm{Mb}$ ) in the OM branch coagulation group was statistically higher compared with those in the sham group (as shown in Table 1; $P<0.01$ ). Figure 3 shows echocardiographic measurements that indicated a significant reduction in LVEF from baseline to 4 weeks after OM branch coagulation (from $53 \% \pm 4.9 \%$ to $33 \% \pm 3.5 \%$, $* * P<0.01$ ). An obvious increase in EDV (from $1.5 \pm 0.7 \mathrm{~m} l$ to $2.0 \pm 0.8 \mathrm{~m} l,{ }^{*} P<0.05$ ) and ESV (from $0.7 \pm 0.1 \mathrm{~m} l$ to $1.5 \pm 0.4 \mathrm{~m} l$, $* P<0.05$ ) was also found. However, these changes did not reach statistical significance in the sham group.

To determine the size of AMI, infarcted tissues were demarcated using Masson's trichrome stain (Fig. 4A). Coagulation of the OM branch reproducibly induced moderate infarcts targeting $32.5 \pm 4.9 \%(n=18)$ of the left ventricle. All animals that received 


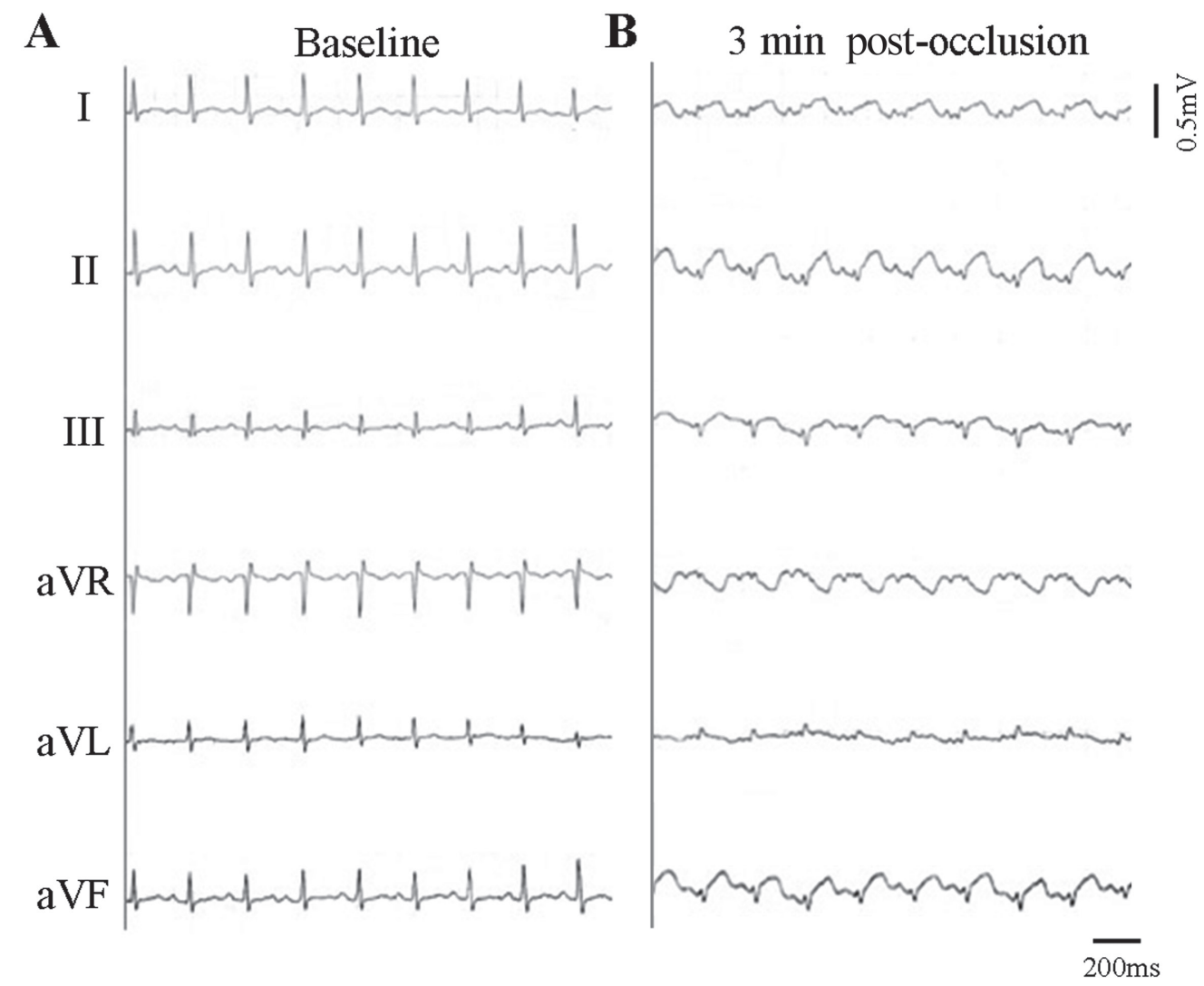

Fig. 2. (A) A representative image of baseline electrocardiography (ECG) of rabbits in the OM branch coagulation group ( $\mathrm{n}=18)$. (B) At 3 min post-coagulation, ECG showed elevated ST segments in V1 $(0.22 \pm 0.07 \mathrm{mV}), \mathrm{V} 2(0.17 \pm 0.05 \mathrm{mV}), \mathrm{V} 3(0.04 \pm 0.01 \mathrm{mV})$ and aVF $(0.07 \pm 0.02$ $\mathrm{mV}$ ) leads. The paper speed in our experiment was $25 \mathrm{~mm} / \mathrm{sec}$.

Table 1. Comparison of the level of AMI-related markers in the blood samples of the sham group $(n=9)$ and OM branch coagulation group $(n=18)$ after surgery

\begin{tabular}{lcc}
\hline \multicolumn{1}{c}{ Myocardial enzyme } & Sham group & OM branch occasion group \\
\hline $\mathrm{hs}-\operatorname{TnI}(n \mathrm{~g} / \mathrm{m} l)$ & $0.14 \pm 0.07$ & $\left.59.15 \pm 32.91^{\mathrm{a}}\right)$ \\
CK-MB $(n \mathrm{~g} / \mathrm{m} l)$ & $0.74 \pm 0.29$ & $\left.2.81 \pm 0.75^{\mathrm{a}}\right)$ \\
Myoglobin $(\mathrm{g} / \mathrm{d} l)$ & $0.04 \pm 0.01$ & $\left.2.45 \pm 0.38^{\mathrm{a}}\right)$ \\
\hline
\end{tabular}

hs-TnT=high-sensitivity troponin $\mathrm{T}, \mathrm{CK}-\mathrm{MB}=$ the $\mathrm{MB}$ isoenzyme of creatine kinase, $\mathrm{Mb}=$ myoglobin. a) $P<0.01$ compared to baseline.

OM branch coagulation showed a transmural extent of infarction mainly targeting the free left ventricular wall, with minimal involvement of the left interventricular septum. Thinness of the left ventricular lateral wall (the thickness decreased from $3.1 \pm$ $0.7 \mathrm{~mm}$ to $2.1 \pm 1.0 \mathrm{~mm}, * P<0.05$ ) and dilatation of the left ventricle (the total left ventricular cross-sectional area increased from $112.2 \pm 16.9 \mathrm{~mm}^{2}$ to $146.4 \pm 15.1 \mathrm{~mm}^{2}, * P<0.01$ ) were also observed in these specimens. In contrast, AMI was not detectable in the heart slices from sham animals. The loss of myocardial cells in the infarcted regions was confirmed by HE staining. Masson's trichrome staining revealed massive collagen deposition in these regions (Fig. 4B).

\section{DISCUSSION}

Large animals, such as the pig [20], rhesus monkey [23] and sheep [12], and smaller animals, such as the rabbit [17], rat [11] and mouse [21], have often been used as experimental animals in AMI-related research. Although experiments using large animals may provide valuable information, the animals are often cost-prohibitive and are difficult to handle and manipulate. Experiments using small animals provide the advantages of lower cost and easy handling. Therefore, small animals have been extensively used in AMI-related research. The rabbit was our choice, because the rabbit heart has minimal collateral arteries; it lacks collateral blood flow after AMI; importantly, there is minimal occurrence of fatal arrhythmia and death after coronary coagulation [1, 2, 14]; both the rabbit and human have the presence of a third coronary artery in common [6]. 
A
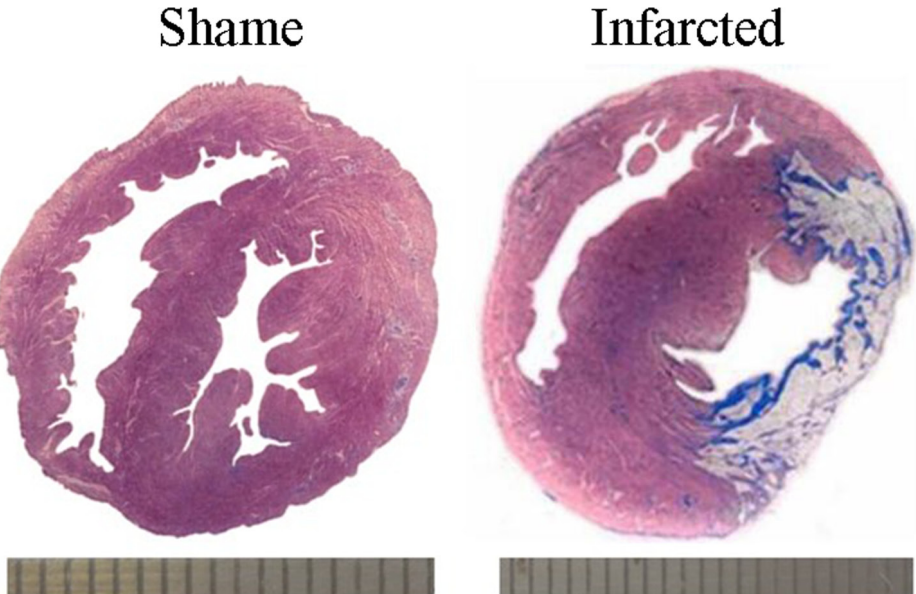

B
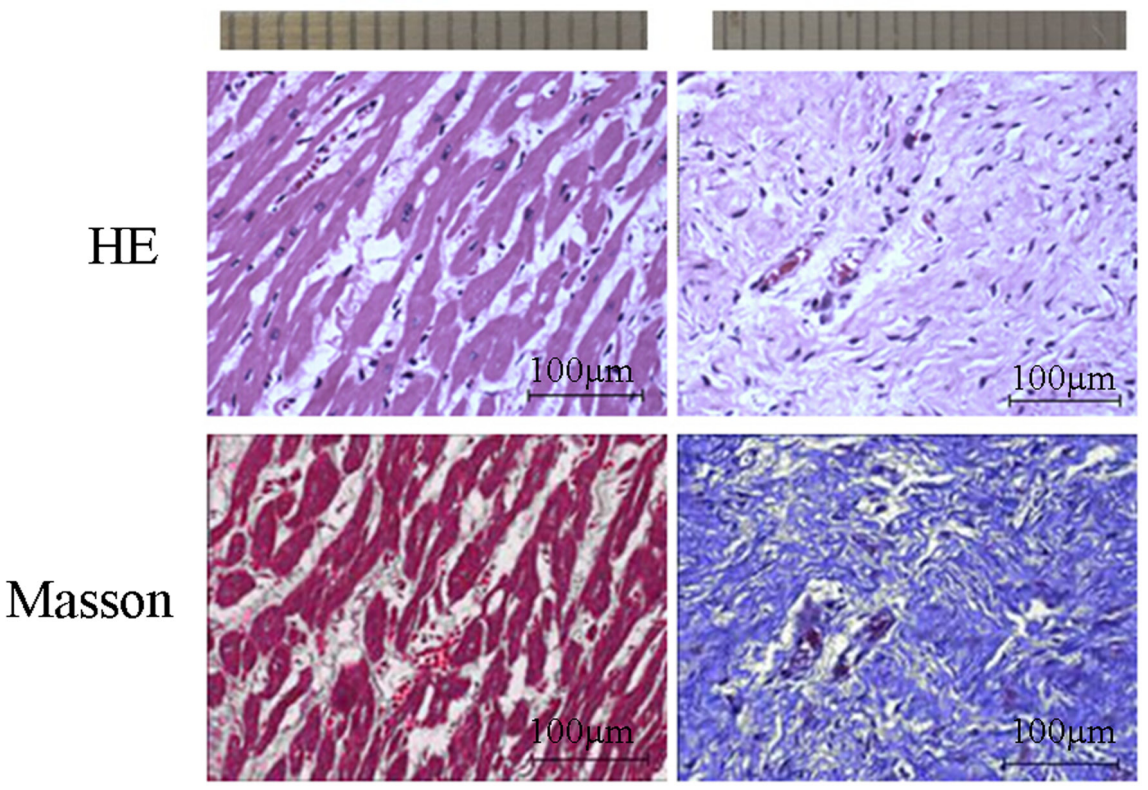

Fig. 3. Representative two-dimensional and corresponding M-mode images of a parasternal short axis view prior to (A) and 4 weeks after electrocoagulation of rabbits in the sham group $(n=9)$ and OM branch coagulation group $(n=18)(B)$. Statistical analysis shows that coagulating the obtuse marginal branch of the left circumflex coronary artery with an electric knife resulted in increased EDV (C) and ESV (D), and decreased LVEF (E). The red vertical line in (A) and (B) indicates the end-diastolic lumen diameter, and the yellow vertical line in (A) and (B) indicates the end-systolic lumen diameter. $\mathrm{EDV}=$ end-diastolic volume, $\mathrm{ESV}=$ end-systolic volume, $\mathrm{EF}=$ ejection fraction. $* P<0.05$ compared to baseline, $* P<0.01$ compared to baseline.

As reported in previous studies, coagulating the OM branch will induce an AMI in patients [7, 13]. In this work, the typical pathological changes of AMI were seen in rabbits after the OM branch was coagulated: ST segment elevation on ECG, increase of myocardial infarction markers in blood samples, cardiac functional damage reflected on echocardiographic images and histological changes in pathological sections. Therefore, to some extent, this rabbit model of AMI shares many properties with human patients.

Previously, the median sternal approach was selected by many researchers to develop AMI models [4, 17, 22]. Using this approach, good exposure of the precordium was obtained, and the pleura were well protected. However, using this approach, the lateral left ventricular wall was not satisfactorily exposed. To create a wider operative field in coagulating the OM branch, a left parasternal longitudinal skin incision was made in this technique. As the mediastinal space in the rabbit is relatively larger than that of other species [25], the chest can thus be opened through a left parasternal approach without the occurrence of pneumothorax. Additionally, the electric knife has been developed as a useful tool with significant coagulation and hemostasis efficacy [9], which was helpful for control of intraoperative blood loss in our study. Hence, the occurrence of hemorrhagic shock could be effectively prevented.

Infarct size is highly dependent on the location of the coagulation site and is a major determinant in cardiac remodeling, hemodynamic dysfunction and mortality [8]. To test the safety and effectiveness of biological therapies, an infarct size induced by ligation of coronary arteries for comparison of experimental groups is required. However, conventional surgical models of AMI established by ligating the LAD after thoracotomy often resulted in infarcts of variable size, because the branches of the LAD are not macroscopically visible [25], which led to "blind" ligation. Previously, an AMI model using a method of ligation of the LAD was used to investigate the curative effect of mesenchymal stem cell seeded into small intestinal submucosa; as it was difficult to 

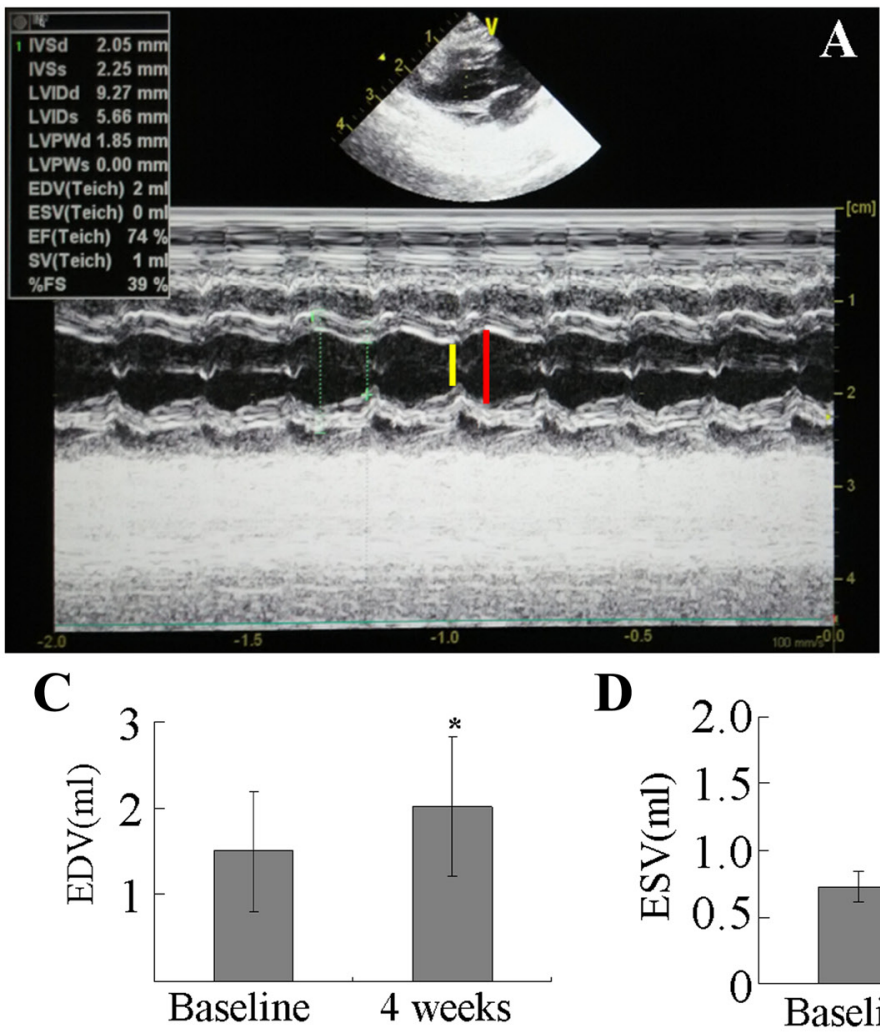

D

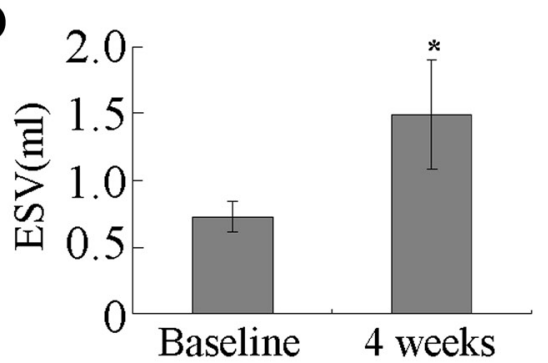

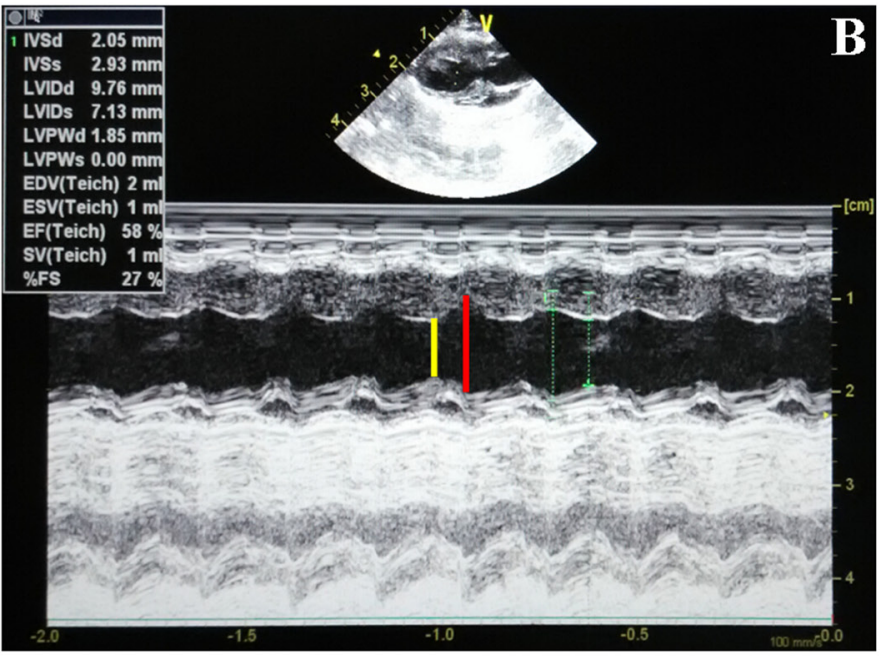

$\mathbf{E}$

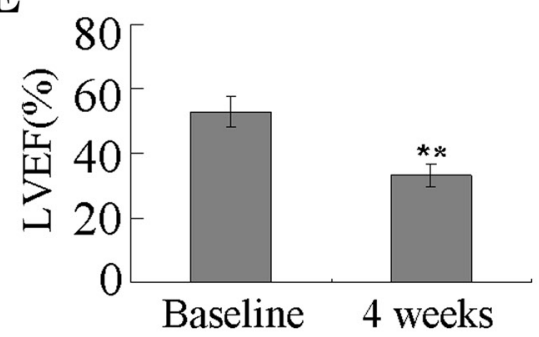

Fig. 4. (A) Representative pathology sections from the sham group $(n=18)$ and the OM branch coagulation group ( $n=9)$ stained with Masson's trichrome at 4 weeks post-thoracotomy. Fibrous scar tissue after transmural infarction is stained pale and blue. Thinning and regional dilation were also observed in the infarct zone. (B) The loss of myocardial cells and the infiltration of leukocytes in the infarcted regions were observed in HE stained sections. Masson's trichrome staining revealed massive collagen (stained blue) deposition in these regions.

identify the course of the LAD and the ideal and correct ligation position, the infarct size was unstable and too many rabbits died [17]. In this new technique, visualization of the OM branch was readily achieved for accurate identification of the ligation site, thus ensuring successful AMI development in rabbits. This method may be suitable for AMI induction in large animals. Before coagulation of the circumflex artery was used for AMI induction in pigs, prohibitively high mortality rates (50\% of the animals) were encountered when the LAD was coagulated [5]. Hence, coagulating the OM branch may be a good choice for AMI induction.

Some limitations in this study should be acknowledged. First, the thoracotomy obviously harmed the rabbits, and its effect on the present results was not investigated. Second, a 4-week follow-up was done to assess the feasibility of this surgical technique for AMI induction, but a longer follow-up period would be better to observe longitudinal pathological changes. Lastly, it is still unknown whether the pathological changes can be reversed.

In conclusion, the left parasternal approach enabled accurate identification of the OM branch with excellent visualization in a rabbit model; the induction of AMI was successfully achieved by coagulating the OM branch with an electric knife. This surgical technique ensured acceptable success rates and low mortality. Therefore, this method of AMI induction in rabbits would be a reliable and reproducible technique for use by researchers.

ACKNOWLEDGMENT. This work was financially supported by a grant (No: 31271049) from the National Science Foundation of China.

\section{REFERENCES}

1. Arcenas, R. F. and Ali, M. I. 2013. Left atrial myxoma: a rare nonatherosclerotic cause of acute myocardial infarction. Case Rep. Cardiol. 2013: 407935. [Medline]

2. Bae, K. R., Lee, Y. S., Kim, B. K., Ha, G. J., Kim, S. Y., Choi, J. Y. and Kim, K. S. 2010. A case of acute myocardial infarction caused by distal embolization of a left main coronary artery thrombus. Korean Circ. J. 40: 46-49. [Medline] [CrossRef]

3. Beitnes, J. O., Lunde, K., Brinchmann, J. E. and Aakhus, S. 2011. Stem cells for cardiac repair in acute myocardial infarction. Expert Rev. Cardiovasc. Ther. 9: 1015-1025. [Medline] [CrossRef]

4. Brehm, C., Schubert, S., Carney, E., Ghodsizad, A., Koerner, M., McCoach, R. and El-Banayosy, A. 2015. Left anterior descending coronary artery blood flow and left ventricular unloading during extracorporeal membrane oxygenation support in a swine model of acute cardiogenic shock. Artif. 
Organs 39: 171-176. [Medline] [CrossRef]

5. Bulbul, A., Can, E., Bulbul, L. G., Cömert, S. and Nuhoglu, A. 2010. Clinical characteristics of neonatal meningomyelocele cases and effect of operation time on mortality and morbidity. Pediatr. Neurosurg. 46: 199-204. [Medline] [CrossRef]

6. Burton, R. A., Schneider, J. E., Bishop, M. J., Hales, P. W., Bollensdorff, C., Robson, M. D., Wong, K. C., Morris, J., Quinn, T. A. and Kohl, P. 2012. Microscopic magnetic resonance imaging reveals high prevalence of third coronary artery in human and rabbit heart. Europace 14 Suppl 5 : v73-v81. [Medline] [CrossRef]

7. Chen, Y., Shao, D. B., Zhang, F. X., Zhang, J., Yuan, W., Man, Y. L., Du, W., Liu, B. X., Wang, D. W., Li, X. R. and Cao, K. J. 2013. Establishment and evaluation of a swine model of acute myocardial infarction and reperfusion-ventricular fibrillation-cardiac arrest using the interventional technique. J. Chin. Med. Assoc. 76: 491-496. [Medline] [CrossRef]

8. Hou, Y., Huang, C., Cai, X., Zhao, J. and Guo, W. 2011. Improvements in the establishment of a rat myocardial infarction model. J. Int. Med. Res. 39: 1284-1292. [Medline] [CrossRef]

9. Jiang, K., Zhu, Y., Zhou, G., Ye, Y., Xie, Q., Yang, X. and Wang, S. 2015. Safe distance between electrotome and recurrent laryngeal nerve: an experimental canine model. Int. J. Clin. Exp. Med. 8: 770-775. [Medline]

10. Katsanos, K., Mitsos, S., Koletsis, E., Bravou, V., Karnabatidis, D., Kolonitsiou, F., Diamantopoulos, A., Dougenis, D. and Siablis, D. 2012. Transauricular embolization of the rabbit coronary artery for experimental myocardial infarction: comparison of a minimally invasive closed-chest model with open-chest surgery. J. Cardiothorac. Surg. 7: 16. [Medline] [CrossRef]

11. Kiss, K., Csonka, C., Pálóczi, J., Pipis, J., Görbe, A., Kocsis, G. F., Murlasits, Z., Sárközy, M., Szűcs, G., Holmes, C. P., Pan, Y., Bhandari, A., Csont, T., Shamloo, M., Woodburn, K. W., Ferdinandy, P. and Bencsik, P. 2016. Novel, selective EPO receptor ligands lacking erythropoietic activity reduce infarct size in acute myocardial infarction in rats. Pharmacol. Res. 113 Pt A: 62-70. [Medline] [CrossRef]

12. Macarthur, J. W. Jr., Cohen, J. E., McGarvey, J. R., Shudo, Y., Patel, J. B., Trubelja, A., Fairman, A. S., Edwards, B. B., Hung, G., Hiesinger, W., Goldstone, A. B., Atluri, P., Wilensky, R. L., Pilla, J. J., Gorman, J. H. 3rd., Gorman, R. C. and Woo, Y. J. 2014. Preclinical evaluation of the engineered stem cell chemokine stromal cell-derived factor $1 \alpha$ analog in a translational ovine myocardial infarction model. Circ. Res. 114: 650-659. [Medline] [CrossRef]

13. Munz, M. R., Faria, M. A., Monteiro, J. R., Aguas, A. P. and Amorim, M. J. 2011. Surgical porcine myocardial infarction model through permanent coronary occlusion. Comp. Med. 61: 445-452. [Medline]

14. Shahzad, K., Cao, L., Ain, Q. T., Waddy, J., Khan, N. and Nekkanti, R. 2013. Postpartum spontaneous dissection of the first obtuse marginal branch of the left circumflex coronary artery causing acute coronary syndrome: a case report and literature review. J. Med. Case Reports 7: 82. [Medline] [CrossRef]

15. Shebuski, R. J. 2002. Utility of point-of-care diagnostic testing in patients with chest pain and suspected acute myocardial infarction. Curr. Opin. Pharmacol. 2: 160-164. [Medline] [CrossRef]

16. Spata, T., Bobek, D., Whitson, B. A., Parthasarathy, S., Mohler, P. J., Higgins, R. S. and Kilic, A. 2013. A nonthoracotomy myocardial infarction model in an ovine using autologous platelets. BioMed. Res. Int. 2013: 938047. [Medline] [CrossRef]

17. Tan, M. Y., Zhi, W., Wei, R. Q., Huang, Y. C., Zhou, K. P., Tan, B., Deng, L., Luo, J. C., Li, X. Q., Xie, H. Q. and Yang, Z. M. 2009. Repair of infarcted myocardium using mesenchymal stem cell seeded small intestinal submucosa in rabbits. Biomaterials 30: 3234-3240. [Medline] [CrossRef]

18. Traverse, J. H. 2012. Using biomaterials to improve the efficacy of cell therapy following acute myocardial infarction. J. Cardiovasc. Transl. Res. 5: 67-72. [Medline] [CrossRef]

19. Uchida, T., Furuno, Y., Tanimoto, A., Toyohira, Y., Arakaki, K., Kina-Tanada, M., Kubota, H., Sakanashi, M., Matsuzaki, T., Noguchi, K., Nakasone, J., Igarashi, T., Ueno, S., Matsushita, M., Ishiuchi, S., Masuzaki, H., Ohya, Y., Yanagihara, N., Shimokawa, H., Otsuji, Y., Tamura, M. and Tsutsui, M. 2014. Development of an experimentally useful model of acute myocardial infarction: $2 / 3$ nephrectomized triple nitric oxide synthases-deficient mouse. J. Mol. Cell. Cardiol. 77: 29-41. [Medline] [CrossRef]

20. Uitterdijk, A., Yetgin, T., te Lintel Hekkert, M., Sneep, S., Krabbendam-Peters, I., van Beusekom, H. M., Fischer, T. M., Cornelussen, R. N., Manintveld, O. C., Merkus, D. and Duncker, D. J. 2015. Vagal nerve stimulation started just prior to reperfusion limits infarct size and no-reflow. Basic Res. Cardiol. 110: 508. [Medline] [CrossRef]

21. Valiente-Alandi, I., Albo-Castellanos, C., Herrero, D., Sanchez, I. and Bernad, A. 2016. Bmi1 (+) cardiac progenitor cells contribute to myocardial repair following acute injury. Stem Cell Res. Ther. 7: 100. [Medline] [CrossRef]

22. van Hout, G. P., Teuben, M. P., Heeres, M., de Maat, S., de Jong, R., Maas, C., Kouwenberg, L. H., Koenderman, L., van Solinge, W. W., de Jager, S. C., Pasterkamp, G. and Hoefer, I. E. 2015. Invasive surgery reduces infarct size and preserves cardiac function in a porcine model of myocardial infarction. J. Cell. Mol. Med. 19: 2655-2663. [Medline] [CrossRef]

23. Wang, Y., Cai, W., Wang, L. and Xia, R. 2016. Evaluate the early changes of myocardial fibers in rhesus monkey during sub-acute stage of myocardial infarction using diffusion tensor magnetic resonance imaging. Magn. Reson. Imaging 34: 391-396. [Medline]

24. Weir, R. A., McMurray, J. J. and Velazquez, E. J. 2006. Epidemiology of heart failure and left ventricular systolic dysfunction after acute myocardial infarction: prevalence, clinical characteristics, and prognostic importance. Am. J. Cardiol. 97 10A: 13F-25F. [Medline] [CrossRef]

25. Yanming, LI, Hong, JIANG, Zhengcong, ZHANG and Fang, WANG. 2013. Anatomical Observation of Coronary Arteries in Rabbit Heart. JOURNAL OF NINGXIA MEDICAL UNIVERSITY. 35: 1099-1102, 1094.

26. Ye, L., Chang, Y. H., Xiong, Q., Zhang, P., Zhang, L., Somasundaram, P., Lepley, M., Swingen, C., Su, L., Wendel, J. S., Guo, J., Jang, A., Rosenbush, D., Greder, L., Dutton, J. R., Zhang, J., Kamp, T. J., Kaufman, D. S., Ge, Y. and Zhang, J. 2014. Cardiac repair in a porcine model of acute myocardial infarction with human induced pluripotent stem cell-derived cardiovascular cells. Cell Stem Cell 15: 750-761. [Medline] [CrossRef] 